\title{
Extracción y caracterización del mucílago de la malva común (malva sylvestris) y del guarumbo (cecropia peltata) para realizar ensayos en la formulación de espumante para la remoción de oro nativo en la minería artesanal
}

Extraction and characterization of the mucilage of the common mallow (malva sylvestris) and guarumbo (cecropia peltata) to carry out trials in the formulation of sparkling wine for the removal of native gold in artisanal mining

Teresita Jackelin Mejía Reinoso. ${ }^{1}$, Javier Ignacio Briones García. ${ }^{2} \&$ Norma del Rocío Toledo Castillo. ${ }^{3}$

Recibido:05-01-2020 / Revisado:22-01-2020 /Aceptado: 03-02-2020/ Publicado: 05-03-2020

\begin{abstract}
DOI: https://doi.org/10.33262/concienciadigital.v3i1.2.1181

The present work is an investigation on the use of Common Mallow (Malva Sylvestris) and Guarumbo (Cecropia Peltata) mucilage, for the formulation of sparkling wine in which the removal of free gold by the flotation method was completed. For this, the hydrophobicity of gold had to be induced with surfactants such as the common detergent and with the help of the viscosity given by the mucilage, the generation of a stable foam was achieved, with a sufficient height where the mineral was concentrated, recovering $72 \%$, compared to $81 \%$ obtained with conventional tests.
\end{abstract}

Keywords: Mucilage, polysaccharide, gold, sparkling, flotation.

\section{Resumen}

El presente trabajo es una investigación sobre la utilización del mucílago de Malva Común (Malva Sylvestris) y del Guarumbo (Cecropia Peltata), para la formulación de espumante en la que se terminó la remoción de oro libre por el método de flotación. Para ello se tuvo que inducir a la hidrofobicidad del oro con surfactantes como el detergente común y con la ayuda de la viscosidad dada por el mucílago, se logró la generación de una espuma estable, con una altura suficiente en donde se concentró el mineral, recuperándose en un $72 \%$, frente al $81 \%$ que se obtiene con ensayos convencionales.

\footnotetext{
${ }^{1}$ Escuela Superior Politécnica de Chimborazo - Sede Morona Santiago,jmejiareinoso@yahoo.com

${ }^{2}$ Escuela Superior Politécnica de Chimborazo - Sede Morona Santiago, javier.briones@espoch.edu.ec

${ }^{3}$ Escuela Superior Politécnica de Chimborazo - Sede Morona Santiago, toledo@espoch.edu.ec
} 
Palabras Clave: Mucílago, polisacárido, oro, espumante, flotación. Introducción

Con el desarrollo de tecnologías más limpias, constantemente se está buscando la utilización de productos, más amigables con el ambiente, que ayuden paulatinamente a ir remplazando sustancias tóxicas que causan problemas ambientales, tanto al ser humano como a su entorno. Es por eso que, en los últimos años, la ciencia se ha volcado al bosque, a la selva, a conocimientos ancestrales, etc.; en búsqueda de sustancias y métodos que sean de fácil acceso, de bajo precio y excelente funcionalidad y que sobre todo que tengan un mínimo impacto en el ambiente y la salud.

Este trabajo de investigación se ha centrado en buscar una alternativa a la obtención de oro nativo o libre que se lava en las riberas de los ríos, por parte grupo de mineros artesanales que es su mayoría son informales y campesinos de la zona, y que en su trabajo diario amalgaman el oro con mercurio o utilizan el proceso de cianuración para la recuperación del preciado metal. Contaminando el afluente que generalmente también les proveer del líquido vital, los peces, y lo más graves a sus propias familias. Pues esto es siempre un tema muy controversial, y que generalmente no es analizado a profundidad.

Un grupo de investigadores de la Escuela Superior Politécnica de Chimborazo, junto con el apoyo de sus autoridades, ha emprendido en la búsqueda de una alternativa, en base a un método de minería como es la flotación espumante, para ello se ha partido desde el análisis de ciertos vegetales como son la (Malva Sylvestris) y el Guarumbo (Cecropia Peltata), para obtención de un polímero, como es el mucílago. Con este producto se ha evaluado la realización de la mejor fórmula, para producir el colector, para luego aplicar a una muestra donde se introdujo experimentalmente una cantidad conocida de oro y determinar si existe una remoción de oro.

\section{Obtención y caracterización del Mucílago}

El mucílago es un polisacárido natural constituido por una fracción neutral compuesta por una cadena principal de $\beta-\mathrm{D}$-xilano y arabinoxilano, donde las cadenas de arabinosa y galactosa están conectada a la posición 2 o 3 de la cadena principal; y otra ácida constituida por una cadena principal de $\alpha-\mathrm{L}$ - ramnopiranosil y residuos del ácido D - galactopiranosiluronico con cadenas de fucosa y galactosa (Oomah et al., 1995).

Tanto la (Malva Sylvestris) como el Guarumbo (Cecropia Peltata), poseen en su estructura mucílagos de alto peso molecular, que forma soluciones viscosas cuando se disuelve en agua, convirtiéndolo en una opción viable para estudiarlo y aplicarlo como hidrocoloide en la 
formulación de agentes viscosos que ayuden a mejorar las espumas, en un sistema de flotación, como el objeto de remover las pequeñas escamas de oro nativo de la arenisca, que ayuden en parte a contrarrestar la utilización de mercurio o cianuro en la obtención de oro en la minería artesanal.

\section{Obtención y recolección de las plantas}

Las plantas malva (Malva Sylvestris) como el Guarumbo (Cecropia Peltata) se recolectó dentro de las instalaciones de la Escuela Superior Politécnica de Chimborazo (ESPOCH) Sede Morona Santiago, estas plantas son endémicas de la zona, de fácil reproducción e invasivas. De las malvas se utilizó las hojas y flores, se limpió, se trituró finamente y se procedió a secar; para luego proceder a la extracción del mucílago. En cuanto al guarumbo, se utilizó la corteza del tallo y las raíces de plantas jóvenes, y se procedió de la misma manera que la malva. Para la extracción del mucílago se utilizó 100g de la planta en 1000ml de agua ionizada.

\section{Métodos de extracción}

Las muestras fueron humectadas en el solvente de extracción (agua destilada ionizada), guardando una relación entre masa del material vegetal y el volumen de agua 1:10.

Cocción: ebullición (se lleva a ebullición y se retira del fuego la mezcla), $0.5 \mathrm{~h}, 1 \mathrm{~h}$ y $2 \mathrm{~h}$; para cada uno de los ensayos se realiza a una temperatura de $95 \pm 2^{\circ} \mathrm{C}$, y se dejó en reposo una hora. Maceración: $1 \mathrm{~h}, 6 \mathrm{~h}, 12 \mathrm{~h}$ y $24 \mathrm{~h}$ a temperatura ambiente.

\section{Ensayo gravimétrico}

Para el ensayo de la extracción del mucílago fue la relación de mezcla en solución mucílago/etanol: 1:1 (v/v), es decir se tomó alícuotas de $25 \mathrm{ml}$ tanto de la muestra como del etanol se agitó vigorosamente y se dejó a que decante el mucílago, luego se filtró y se llevó a secarse en una estufa a $35^{\circ} \mathrm{C}$ por $24 \mathrm{~h}$, para posteriormente pesar.

\section{Resultados}

\begin{tabular}{|c|c|}
\hline \multicolumn{2}{|c|}{$\begin{array}{l}\text { nucligos de malva extraid } \\
\text { Malva }\end{array}$} \\
\hline $\begin{array}{l}\text { Tiempo de } \\
\text { cocción (h) } \\
\text { Ebullición (0) }\end{array}$ & $\begin{array}{c}\text { Masa de mucilago } \\
\text { obtenido (g) } \\
4.2\end{array}$ \\
\hline 0.5 & 5.6 \\
\hline 1 & 5.8 \\
\hline 2 & 5.8 \\
\hline
\end{tabular}




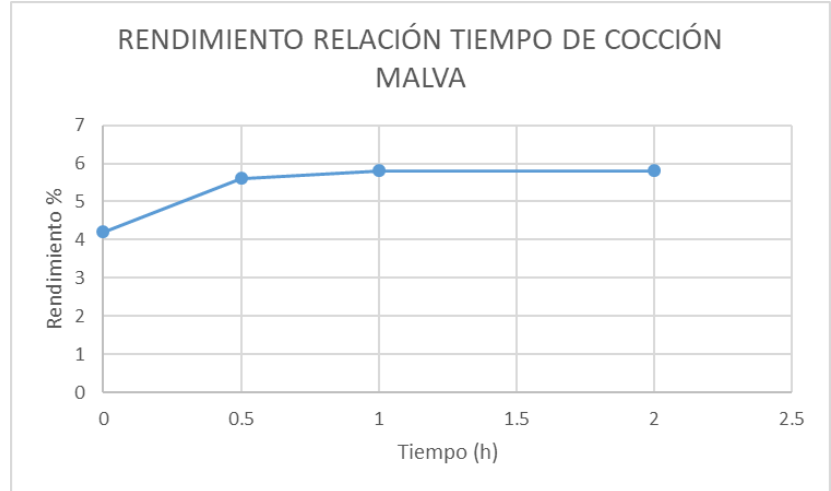

\section{Figura 1}

Rendimiento del mucílago de la malva vs tiempo de cocción.

( 0 en la abscisa $x$ significa ebullición)

Elaborado por: Grupo de Investigación

\section{Tabla 2}

Resultados de g/L de mucílago de Guarumbo extraídos por cocción.

\begin{tabular}{cc}
\hline \multicolumn{2}{c}{ Guarumbo } \\
\hline $\begin{array}{c}\text { Tiempo de cocción } \\
\text { (h) }\end{array}$ & $\begin{array}{c}\text { Masa de mucílago } \\
\text { obtenido(g) }\end{array}$ \\
Ebullición (0) & 1.1 \\
0.5 & 1.9 \\
1 & 2 \\
2 & 2
\end{tabular}

Elaborado por: Grupo de Investigación

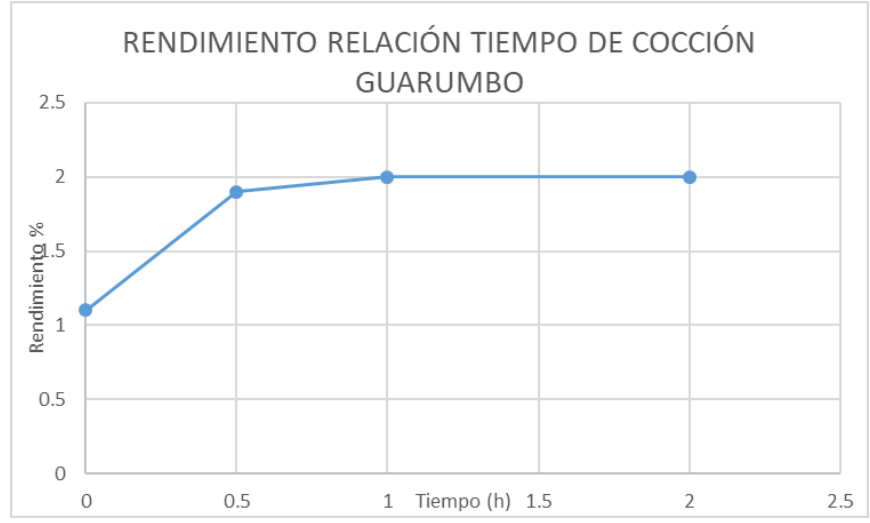

Figura 2

Rendimiento del mucílago en el guarumbo vs tiempo de cocción.

(0 en la abscisa $x$ significa ebullición) 
Tabla 3

Resultado tiempo de maceración

\begin{tabular}{cc}
\hline \multicolumn{2}{c}{ Malva } \\
\hline Tiempo de maceración (h) & Masa de mucilago obtenido \\
& $\mathbf{( g )}$ \\
6 & 2.2 \\
12 & 2.6 \\
24 & 3.8 \\
& 5.2
\end{tabular}

Elaborado por: Grupo de Investigación.

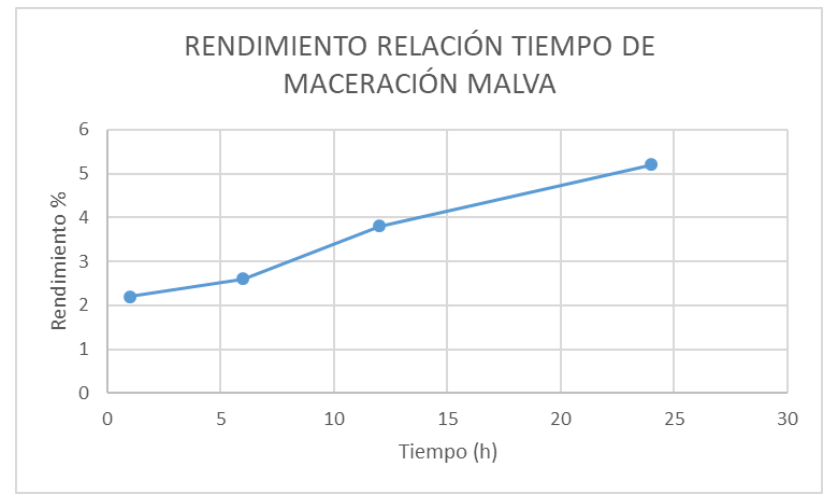

Figura 3

Rendimiento del mucílago de la malva vs tiempo de maceración.

(0 en la abscisa $x$ significa ebullición)

Elaborado por: Grupo de Investigación

\section{Tabla 4}

Resultados de g/L de mucíligos de guarumbo extraídos por maceración.

\begin{tabular}{cc}
\hline \multicolumn{2}{c}{ Guarumbo } \\
\hline Tiempo de maceración (h) & $\begin{array}{c}\text { Masa de mucílago } \\
\text { obtenido }(\mathbf{g})\end{array}$ \\
1 & 0.6 \\
6 & 1.1 \\
12 & 1.7 \\
24 & 1.9
\end{tabular}

Elaborado por: Grupo de Investigación 


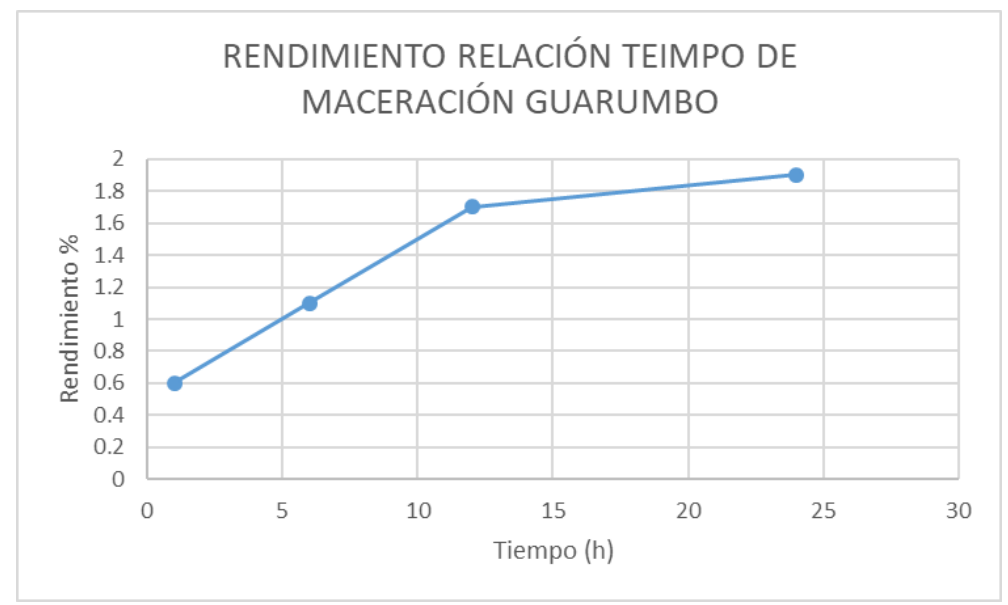

Figura 4

Rendimiento del mucílago del guarumbo vs tiempo de maceración. (0 en la abscisa $x$ significa ebullición)

Elaborado por: Grupo de Investigación

\section{Formulaciones espumantes}

La espuma es un coloide en donde un gas generalmente aire se encuentra atrapado en un sistema líquido. Las espumas generalmente cuentan con una pequeñísima cantidad de líquido conformando las paredes de las burbujas conocidas como "lamelas" y, por ser un sistema coloidal, cuenta con una alta viscosidad. Para generar una formulación espumante, es necesario contar al menos dos componentes principales: un tensoactivo capaz de generar las lamelas, y un agente viscosificante (mucílago) que ayudará a evitar que la lamela drene.

Para nuestra investigación, es importante analizar la cantidad de espuma que puede generar el colector, este componente fue analizado por medio de la altura de espuma. Esta es de gran impacto en la selectividad del proceso de flotación. Laskowski, J.S. y Melo, F. (2005). No existe una regla general para la determinación de la altura de espuma. Yianatos, J.B., Bergh (1994), pero como parámetro de operación, en una columna de flotación se usan alturas que varían entre 1 a $2 \mathrm{~m}$, mientras que, en escala piloto, estos valores se sitúan entre 0,2 y 1,0 m.

\section{Metodología}

Se utilizó una solución base de $500 \mathrm{ml}$ de agua ionizada en el cual se adiciona $0.5 \mathrm{~g}$ de detergente común. De esta solución base se tomó $10 \mathrm{ml}$ para luego ir añadiendo diferentes $\%$ de peso de los dos tipos de mucílagos extraídos y se incorporó una corriente de aire, para generar espuma. Posteriormente se procedió a medir la altura de espuma generada en cada uno de los ensayos. 
Resultados

Tabla 5

Resultado de las pruebas de estabilidad de espuma

\begin{tabular}{lccc}
\hline $\begin{array}{c}\text { TIPO DE } \\
\text { MUCÍLAGO }\end{array}$ & $\begin{array}{c}\text { MUCÍLAGO } \\
\text { (\% peso) }\end{array}$ & $\begin{array}{c}\text { ESPUMA } \\
\text { (cm) }\end{array}$ & PH \\
\hline BLANCO & 0.00 & 10 & 7.8 \\
GUARUMBO & 0.25 & 12 & 7.8 \\
GUARUMBO & 0.50 & 35 & 8 \\
GUARUMBO & 0.75 & 42 & 8 \\
GUARUMBO & 1.00 & 48 & 8.4 \\
MALVA & 0.25 & 20 & 7.7 \\
MALVA & 0.50 & 35 & 7.9 \\
MALVA & 0.75 & 53 & 8.4 \\
MALVA & 1.00 & 60 & 8.4 \\
\hline
\end{tabular}

Elaborado por: Grupo de Investigación

\section{Ensayos de flotación}

La flotación, es un proceso fisicoquímico que consiste en separar partículas "hidrófobas" (repelentes o sin afinidad química con el agua) de partícula "hidrofílicas"(afines químicamente con el agua), asistida con inyección de burbujas de aire. Bustamante (2008). Este proceso es uno de los más importantes para la recuperación de oro y metales valiosos. Su principal restricción es recuperar partículas de material grueso (intervalo de 400 - $250 \mu \mathrm{m}$ ).

La forma de la partícula juega un papel importante en la recuperación de oro libre por medio de la flotación espumante, generalmente en los ríos se encuentra oro en forma de pequeñas escamas. Esta forma aplanada desarrolla una mayor área superficial y pueden interactuar de manera más eficiente con el colector y la adsorción podría ser mucho más rápida comparada con pepitas pequeñas o partículas redondeadas, por lo que este factor tiene importancia en la flotación según Chyssoulis et al. L. Chryssoulis S. (2003). Por lo tanto, los factores que pueden afectar la flotabilidad del oro libre. C. J. van Oss. (2007), A. J. Teague (1999), son: el tamaño, la forma y la composición de la partícula, junto con la presencia de contaminantes superficiales, el tipo de colector y la geometría de las celdas.

Hay minerales que son hidrófobos por naturaleza como (talco, azufre, molibdenita, grafito), pero la gran mayoría, entre los que se encuentra el oro, son hidrófilos, se humectan en el medio acuoso, al establecer enlaces superficiales con los grupos iónicos, lo que se conoce como 
adsorción de agua o humectación S. Castro and J. A. García. (2003). Para que estas partículas de oro se vuelvan hidrófobas es necesario adicionar una sustancia tensoactiva que generen la reducción de la tensión superficial de la interface sólido-líquido y permita la adsorción espontánea de burbujas de aire para que el sistema partícula-burbujas ascienda hasta la superficie, en la industria minera se obtiene la hidrofobicidad del oro cuando se somete a la acción de los tiocolectores como xantatos disueltos en la solución acuosa.

Otro factor importante que hay que tomar en cuenta es el tamaño de la partícula que tiene un gran efecto sobre la recuperación de oro mediante flotación debido a su alta densidad. La flotación es efectiva para partículas de oro en el intervalo de 20-200 micrones. Para tamaños más finos la selectividad del oro decrece debido a la flotación de minerales ganga. Para tamaños de partícula más gruesos la flotación debe realizarse con altas densidades de la mezcla (35\% sólidos) dado que esto reduce el tamaño de partícula de la sedimentación. Castro S.J, (2003). En esta investigación se procedió hacer una serie de formulaciones para la flotación espumante directa como alternativa para el uso de mercurio, en la recuperación de oro nativo en afluentes de agua dulce. Para los ensayos pertinente se intentó imitar a una celda tipo "flash.", en la que se hicieron los análisis pertinentes.

\section{Ensayo y resultados}

Se construyó una celda con características tipo "flash.”, de $60 \mathrm{~cm}$ de alto, el volumen utilizado en la celda fue de $500 \mathrm{ml}$ del espumante $(500 \mathrm{ml}$ de agua ionizada, $0.5 \mathrm{~g}$ de detergente y $7.5 \mathrm{~g}$ de mucílago de malva), se introdujo una muestra previamente pesada de oro de aluvión con una granulometría en el orden de 20 a 200 micras (6g). Se procedió a introducir una corriente de aire por en un tiempo de 20 minutos, generando espuma abundante.

Se fue recogiendo la espuma donde se encontraba el concentrado del mineral precioso y luego se procedió a pesar. Se realizaron cinco repeticiones, con un rendimiento promedio del $72 \%$ frente al tratamiento convencional donde se tiene una recuperación de hasta el $81 \%$.

\section{Conclusiones}

Estas dos plantas son endémicas de la Amazonía encontrándose en cualquier lugar, siendo muy fácil su recolección.

Para la extracción del mucílago: para la malva, la técnica de cocción por una hora con un reposo de media hora, fue la más óptima, ya que tiene un rendimiento del 5.8\% y para el guarumbo igualmente fue la técnica de cocción por una hora con un reposo de media hora, con un rendimiento del $2.0 \%$. Se determinó por lo tanto que la malva contiene más mucílago que el guarumbo. 
Para las pruebas de formulación del espumante se utilizó el mucílago de las dos plantas por separado.

El mucílago de la malva es más fácil extraer ya que este compuesto se encuentra en prácticamente toda la planta. En el gurumbo la extracción del mucílago de las hojas es casi imposible determinar por estos métodos empleaos por lo que se descartó y solo se utilizó para el análisis las cortezas de los tallos y raíces.

El mucílago en general, incrementó la viscosidad provocando una mejor formación de espuma, provocando una mejor estabilidad.

Se observó que conforme se aumenta el mucílago en la solución de detergente, mejores resultados se obtuvieron en la formulación del espumante, siendo el más óptimo el de la malva con un porcentaje en peso del $1.00 \%$.

También se observó que en la muestra se incrementaba el pH, obteniendo hasta un pH de 8.4 con el $1.00 \%$ del mucílago de la malva, aunque con el guarumbo igualmente se obtuvo ese mismo $\mathrm{pH}$.

Para la formulación del agente espumante se utilizó el mucílago de la malva con el $1.00 \%$ en peso, porque dio una altura de espuma estable de $50 \mathrm{~cm}$ con $10 \mathrm{~s} 10 \mathrm{ml}$.

Se descartó el mucílago del guarumbo, primeramente, porque es más difícil su extracción y la altura provocada por su espuma fue superado por el mucílago de la malva.

La flotación espumante se puede definir brevemente como un proceso de concentración que aprovecha las diferencias en las propiedades físico-químicas superficiales de las partículas minerales.

Se observa que esta formulación si remueve el oro, siembre y cundo este esté en forma libre y con una geometría de escamas.

Se logró una eficiencia del $72 \%$, pero si se tecnifica más posiblemente este rendimiento subo. El método de flotación directa es una buena alternativa para la recuperación de oro que podría sustituir al proceso de amalgamación tradicional.

\section{Recomendaciones}

Analizar otras técnicas, para mejor la obtención del mucílago.

Para realizar el proceso de flotación espumante, es necesario garantizar la hidrofobicidad de las partículas a flotar, que le permite la adherencia de la partícula a la burbuja.

Utilizar el mucílago con sulfuros como: la piritas, calcopiritas, galena y esfalerita, para garantizar la hidrofobicidad natural de las partículas de oro, y el proceso de flotación sea más óptimo. 


\section{Bibliografía}

A. J. Teague, J. S. J. Van Deventer, and C. Swaminathan, "A conceptual model for gold flotation,” Minerals Engineering, vol. 12, no. 9, pp. 1001-1019, Sep. 1999.

Balke D, Diosady L. Rapid aqueous extraction of mucilage from whole white mustard seed. Food Research International. 2000; 33:347-56.

Bustamante R. M.O., Gaviria C. A.C., Restrepo B.O.J. Notas de la asignatura Concentración de Minerales. Facultad de Minas, Universidad Nacional de Colombia. 111 pig. 2008.

C. J. van Oss, "Development and applications of the interfacial tension between water and organic or biological surfaces," Colloids and surfaces. B, Biointerfaces, vol. 54, no. 1, pp. 2-9, Jan. 2007.

Hindustan A A, Sreeramulu J, Sreenivasulu R, Suma Padmaja B, Narasimha Reddy M. Fabrication of glimepiride Hibiscus esculentus fruit mucilage and povidone sustained release matrix tablets: In vitro evaluation. Der Pharmacia Sinica. 2011;2(2):91-100.

Laskowski, J.S. y Melo, F. (2005). Fundamental properties of flotation frothers and their effect on flotation. Journal of Minerals Engineering, 766-773.

Lih-Shiuh L, Hui-Yuan L. Chemical compositions and some physical properties of the water and alkali-extracted mucilage from the young fronds of Asplenium australasicum (J. Sm.) Hook. Food Hydrocolloids. 2012; 26:344-9.

L. Chryssoulis S, D. Venter, and S. Dimov, "On the floatability of gold grains. 35th Annual Meeting of the Canadian Mineral Processors.pdf." Canada, pp. 455-472, 2003.

Malviya R, Srivastava P, Kulkarni GT. Applications of Mucilages in Drug Delivery-A Review. Advances Biological Research. 2011;5(1):1-7.

Marín M, Acevedo J, Tamez M, Nevero J. Proceso de obtención del mucílago de Salvia hispánica L. México: Instituto tecnológico de estudios superiores de Monterrey, Nuevo León. WO 2008/044908 A2; 2008.

M. C. Fuerstenau and K. N. Han, Principles of Mineral Processing.pdf. Littleton: Society for Mining Metallurgy and Exploration Inc., 2003, p. 573.

Oomah BD, Kenaschuk EO, Mazza G (1995) Phenolic acids in flaxseed. J Agriculture Food Chem 43:2016-2019

Pareek V, Singh M, Bhat ZA, Singh P, Kumar D, Sheela S. Studies on mucilage of Basella alba Linn, J Pharmacy Research. 2010;3(8):1892-4. 
Pérez P. Mucílago pulverizado obtenido a partir de la cáscara de cacao. Una alternativa en la clarificación de los jugos en la industria de la panela. Manizales, Colombia; 2004. p. 15.

Sepúlveda E, Sáenz C, Aliaga E, Aceituno C. Extraction and characterization of mucilage in Opuntia spp. J Arid Environments. 2007; 68:534-45. 4. BDN. Hidrocoloides; 2008. [citado Sep. 2012]. [3 pantallas].

Shah BN, Seth AK, Nayak BS. Microwave Assisted Isolation of Mucilage from the Fruits of Richosanthes dioica, FABAD J Pharm Sci. 2010;33:131-4.

S. Castro and J. A. García, Flotación Fundamentos y Aplicaciones.pdf, Primera. Santiago de Chile: Universidad de Concepción, Facultad de Ingeniería, Departamento de Ingeniería Metalúrgica, 2003, p. 182.

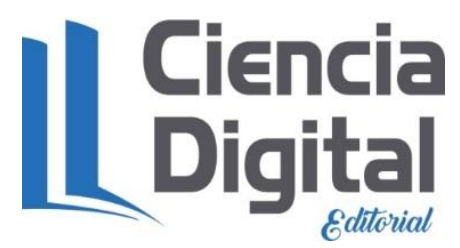




\section{PARA CITAR EL ARTÍCULO INDEXADO}

Mejía Reinoso, T. J., Briones García, J. I., \& Toledo Castillo, N. del R. (2020). Extracción y caracterización del mucílago de la malva común (malva sylvestris) y del guarumbo (cecropia peltata) para realizar ensayos en la formulación de espumante para la remoción de oro nativo en la minería artesanal. ConcienciaDigital, 3(1.2), 92-103.

https://doi.org/10.33262/concienciadigital.v3i1.2.1181

\section{Ciencia \\ LDigital}

El artículo que se publica es de exclusiva responsabilidad de los autores y no necesariamente reflejan el pensamiento de la Revista Conciencia Digital.

El artículo queda en propiedad de la revista y, por tanto, su publicación parcial y/o total en otro medio tiene que ser autorizado por el director de la Revista Conciencia Digital.

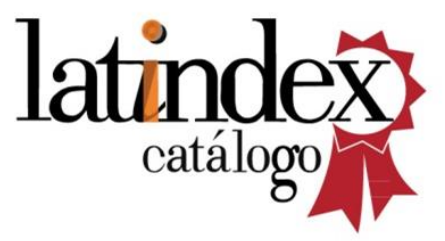

